\title{
A Práxis do Enfermeiro na Assistência Prestada à Saúde do Idoso Usuário do Serviço Hospitalar Oncológico
}

Andreane Natália Pereira de Almeida Monteiro ${ }^{1}$; Maria Amanda Lima Batista ${ }^{2}$; Juliane da Silva Pereira ${ }^{3}$; Luciclaudio da Silva Barbosa ${ }^{4}$; Claudia Daniele Barros Leite-Salgueiro ${ }^{5}$; Luzineide Lobato ${ }^{6}$

\begin{abstract}
Resumo: O presente estudo é uma revisão integrativa sobre a prática de enfermeiros na assistência à saúde do idoso usuário de serviço hospitalar em oncologia. Objetivo: $\mathrm{O}$ estudo buscou demonstrar o perfil dos trabalhos publicados em âmbito nacional, no período de 2011 a 2016, com o levantamento feito a partir do Portal Regional da Biblioteca Virtual de Saúde (BVS), direcionando para periódicos indexados nas bases LILACS e SciELO. Método: A amostra foi apresentada em um quadro sinótico com a distribuição dos artigos analisados. Conclusão: Os autores encontraram ampla discussão na literatura, principalmente considerando-se o modelo humanista de assistência, no qual enfermeiro poderá ouvir objetivamente o indivíduo, identificar suas necessidades e ajudá-lo a encontrar soluções a partir do aumento das informações de sua própria atividade mental, instrumentalizando-o para o agir. Portanto, na consideração de situações em que a vida dos pacientes é permeada por limiares críticos, os profissionais devem estabelecer uma assistência baseada nos princípios éticos e agir de forma reflexiva, a fim de garantir um cuidado efetivo e humanizado.
\end{abstract}

Descritores: Idoso; cuidado de enfermagem; serviço hospitalar de oncologia.

\section{The Practice of the Nurse in the Health Care Assistance of the Older User of the Oncological Hospital Service}

\begin{abstract}
The present study is an integrative review on the practice of nurses in the health care of elderly users of hospital services in oncology. Objective: The study sought to demonstrate the profile of the works published in the national context, from 2011 to 2016, with a survey made from the Regional Portal of the Virtual Health Library (VHL), targeting indexed journals in the LILACS and SciELO databases. Method: The sample was presented in a synoptic table with the distribution of the articles analyzed. Conclusion: The authors found a wide discussion in the literature, especially considering the humanistic model of care, in which nurses can objectively listen to the individual, identify their needs and help them find solutions by increasing the information of their own mental activity, instrumentalizing it to act. Therefore, in considering situations in which patients' lives are permeated by critical thresholds, practitioners should establish care based on ethical principles and act reflexively to ensure effective and humane care.
\end{abstract}

Keywords: Elderly; nursing care; hospital oncology service.

\footnotetext{
${ }^{1}$ Enfermeira formada pelo Instituto Federal de Educação, Ciência e Tecnologia de Pernambuco (IFPE) - Campus Pesqueira. Pesquisadora Colaboradora de Projetos de Pesquisa e Extensão, na linha de Cuidado à Pessoa Idosa, do Instituto Federal de Educação, Ciência e Tecnologia de Pernambuco (IFPE) - Campus Pesqueira. Contato: andreanenathalia@ outlook.com .

${ }^{2}$ Enfermeira formada pelo Instituto Federal de Educação, Ciência e Tecnologia de Pernambuco (IFPE) - Campus Pesqueira. Residente em Saúde da Família /Atenção Básica. Contato: amandalima434@ gmail.com .

${ }^{3}$ Enfermeira formada pelo Instituto Federal de Educação, Ciência e Tecnologia de Pernambuco (IFPE) - Campus Pesqueira. Pesquisadora Colaboradora de Projetos de Pesquisa e Extensão, na linha de Cuidado à Pessoa Idosa, do Instituto Federal de Educação, Ciência e Tecnologia de Pernambuco (IFPE) - Campus Pesqueira. Assistente de saúde da Prefeitura Municipal de Pesqueira-Pe. Contato: julianedasilvapereira@gmail.com

${ }^{4}$ Mestre em Recursos Naturais pela Universidade Federal de Campina Grande (UFCG). Biólogo formado pela Universidade Estadual da Paraíba (UEPB). Docente do Curso de Bacharelado em Enfermagem do Instituto Federal de Educação, Ciência e Tecnologia de Pernambuco (IFPE) - Campus Pesqueira. Coordenador dos laboratórios de morfofisiologias dos processos vitais em enfermagem do Instituto Federal de Educação, Ciência e Tecnologia de Pernambuco (IFPE)-Campus Pesqueira. Contato: luciclaudio@pesqueira.ifpe.edu.br.

${ }^{5}$ Doutora em Psicologia Clínica pela Universidade Católica de Pernambuco (UNICAP), Mestra em Ciências da Saúde pela Universidade de Pernambuco (UPE), Docente do curso Bacharelado em Enfermagem do Instituto Federal de Educação, Ciência e Tecnologia de Pernambuco (IFPE) - Campus Pesqueira. Contato: claudia.leite@ pesqueira.ifpe.edu.br.

${ }^{6}$ Biológa (UFRN), Docente e Pesquisadora do Instituto Federal de Educação, Ciência e Tecnologia de Pernambuco (IFPE) -

Campus Pesqueira. Mestra em Psicobiologia pela Universidade Federal do Rio Grande do Norte (UFRN). Recife (PE), Brasil. Email: luzineidelobato@gmail.com
} 


\section{Introdução}

O Brasil é considerado um país de população envelhecida, uma vez que os dados do censo de 2010 retratavam que o número de idosos na população já representava 11\%, e, para ser considerado um país de população envelhecida, a proporção de idosos alcança $7 \%$, com tendência a aumentar (IBGE, 2010).

Juntamente com essa transição demográfica, observa-se também a transição epidemiológica, esta, caracterizada pela redução da morbimortalidade por doenças infecto-parasitárias e pelo aumento desta por doenças crônicas. As doenças crônicas são em geral incuráveis, demandam de um tratamento contínuo e possuem complicações que podem resultar em incapacidades funcionais (ALMEIDA, AGUIAR, 2011).

Em 2007, ocorreram 2.300.951 internações de idosos em hospitais públicos ou credenciados ao SUS. As principais causas foram as doenças do aparelho circulatório, que totalizaram $28 \%$, doenças do aparelho respiratório que representaram $17 \%$, doenças do aparelho digestivo, que alcançaram 10\% e neoplasias que chegaram a 9\% (BRASIL, 2008).

Dados consolidam que o Instituto Nacional do Câncer (INCA) estimou, em 2011, que no Brasil ocorreriam cerca de 489.270 novos casos de câncer, sendo os tipos mais incidentes os cânceres de próstata e de pulmão nos homens e os cânceres de mama e do colo do útero nas mulheres.

O incremento das taxas de incidência por neoplasias no Brasil pode ser explicado diante da evolução, aumento da qualidade dos métodos diagnósticos e dos dados advindos dos Sistemas de Informação, além da expectativa de vida aumentada (BRASIL, 2012).

A complexidade e a capilaridade do tratamento de câncer requerem habilidades tanto técnico-científicas, como de relações interpessoais e espirituais. A informação adjunta à afetividade, comunicação, sinceridade e empatia, formam matéria-prima para o cuidado, os quais estarão influenciando o desenvolvimento da assistência prestada ao paciente oncológico (PETERSON, CARVALHO, 2011).

Considera-se que os profissionais de saúde, em especial os que trabalham com oncologia, constantemente experimentam situações fadigantes ao mesmo tempo em que são portadores de notícias ruins. Tal situação muitas vezes, podem levar o trabalhador ao estresse e ao sentimento de solidão, que podem ser diminuídos com diálogos que são verdadeiros utensílios de promoção da saúde (BRASIL, 2010). 
A equipe de enfermagem, que atende aos pacientes oncológicos com prognóstico sem possibilidade de cura, precisa ter, além de habilidade técnica para prestar cuidados físicos, sensibilidade nos aspectos psicológicos envolvidos, pautado na ética e na humanização (PINTO et al, 2011). Muitas vezes as neoplasias estão associadas a sofrimento e dor, o que acarreta ao paciente grande instabilidade emocional durante a vivência da doença e de seu tratamento (SALIMENA et al., 2013).

Desse modo, o conhecimento dos profissionais de enfermagem é efetivo na promoção proporcionar da melhoria na qualidade de vida do paciente com câncer, considernado características como a individualidade, as crenças, valores, singularidade, estilo de vida, dentre outras (ALVES et al., 2011).

Ressalta-se que àqueles profissionais que conduzem o tratamento oncológico devem indicar que o sofrimento do paciente oncológico, acirrado pela dor e pelos efeitos do tratamento, têm vários aspectos e repercussões capilarizadas pela sua família e com a equipe profissional (RENNÓ, CAMPOS, 2014).

Em razão à dificuldade de cuidar de pacientes oncológicos, evidencia-se o imperativo de desenvolver estratégias de enfrentamento, ponderando acerca dos aspectos éticos enredados nas distintas situações e nas analogias dos contextos do cuidado, enfrentamento este que pode ser acentuado como um contíguo de contornos comportamentais que o indivíduo emite, quando da vivência de uma situação de estresse, na tentativa de modificar o ambiente, reduzindo ou minimizando seu caráter aversivo (FURTADO et al., 2009; AMADOR et al., 2011).

Neste sentido, acredita-se que averiguar, através de revisão integrativa da literatura, a práxis do enfermeiro na assistência prestada à saúde do idoso usuário do serviço hospitalar oncológico; apanhar o "estado da arte" referente à temática; investigar a assistência prestada pelo enfermeiro à saúde do idoso usuário do serviço hospitalar oncológico e problematizar, acerca dos benefícios do cuidado do profissional de enfermagem, no que tange às searas de qualificação e humanização, reconhecidos pela idoso usuário do serviço hospitalar oncológico, sejam de extrema relevância. Todos estes foram os objetivos do presente artigo. 


\section{Método}

O presente estudo é uma revisão integrativa da literatura, referente à produção do conhecimento sobre a práxis do enfermeiro, na assistência à saúde do idoso usuário do serviço hospitalar oncológico. Tal método oportuniza o exame de estdos científicos de modo sistemático e amplo, favorecendo assim a caracterização e a divulgação do conhecimento produzido (MOREIRA et al., 2015), como também propicia o sumário do estado do conhecimento de um dado tema, conhecido também como estado da arte, possibilitando a identificação do lapso que há no conhecimento, precisando ser preenchidos com a realização de novas pesquisas (POLIT E BECK, 2011). O método defende a síntese de vários estudos publicados e permite conclusões gerais acerca de um campo particular de estudo (MOREIRA et al., 2015).

Essa modalidade de pesquisa é norteada por uma trajetória metodológica composto por seis fases distintas, destacando-se: 1-estabelecimento do problema da revisão (elaboração da pergunta norteadora; 2-estabelecimento de descritores e dos critérios para inclusão/exclusão de artigos); 3-amostragem (seleção dos artigos); 4categorização do estudo; 5-definição das informações a serem extraídas dos trabalhos revisados; 5- descrição d análise e discussão a respeito das tecnologias utilizadas/desenvolvidas e 6-síntese do conhecimento dos artigos analisados ou apresentação da revisão integrativa, na situação presente, através de quadro sinótico evidenciando as principais características (MOREIRA et al., 2015).

Com a finalidade de operacionalização dos procedimentos do presente estudo de revisão integrativa da literatura, inicialmente, identificou-se o tema de interesse, depois, a pesquisa foi conduzida partindo-se da seguinte questão norteadora: Qual a Assistência do Enfermeiro à Saúde da Mulher Idosa residente em ILPI difundida em periódicos online, no período de 2010 a 2015 ?

Para a especificação das publicações que compuseram a revisão integrativa deste estudo, realizou-se uma busca online, com o levantamento a partir da Biblioteca Virtual de Saúde - Portal Regional (BVS) e posterior endereçamento à Scientific Electronic Library Online SciELO e Literatura Latino-Americana e do Caribe em Ciências da Saúde - LILACS, nos meses de setembro e outubro de 2017. Para tanto, foram empregados os seguintes descritores em Ciências da Saúde (DeCS): idoso, cuidado de enfermagem e serviço hospitalar de oncologia. 
O estudo foi constituído por 39 publicações atinentes à temática investigada, disponibilizadas em periódicos disponibilizados online, todos, constituintes da amostra, considerando-se os seguintes critérios de inclusão previamente estabelecidos: artigos publicados em português, disponíveis na íntegra, no período de 2011 a 2016, na modalidade artigo científico original e estudos de revisão. Em relação aos critérios de exclusão, levaram-se em conta: artigos em duplicidade, publicados em idiomas estrangeiros ou que não estivessem no período determinado pelos autores, também, os que não abordavam diretamente à temática proposta.

No tocante aos critérios utilizados para a classificação dos artigos na modalidade revisão, que, trata-se de uma pesquisa realizada mediante material previamente analisado. Convém destacar que a publicação original, versa sobre aquela que apresenta resultados conclusivos.

A fim de viabilizar a coleta dos dados, foi elaborado um quadro sinótico contendo as seguintes informações: título, autor (es), ano de publicação, modalidade do artigo, área do periódico e objetivos do artigo.

\section{Resultados e Discussão}

A seguir, apresenta-se a Distribuição dos artigos (n), segundo ano, título, autores, modalidade de artigo, área da revista e objetivos das publicações selecionadas. 


\begin{tabular}{|c|c|c|c|c|c|}
\hline Ano & Título & Autor (es) & Modalidade & $\begin{array}{l}\text { Área } \\
\text { Revista }\end{array}$ & Objetivos \\
\hline 2016 & $\begin{array}{lr}\text { Estratégias } & \text { de } \\
\text { enfrentamento } & \text { por } \\
\text { enfermeiros } & \text { da } \\
\text { oncologia na alta } \\
\text { complexidade. }\end{array}$ & $\begin{array}{l}\text { Kely Regina da Luzi, Mara } \\
\text { Ambrosina de Oliveira Vargas, } \\
\text { Edison Luiz Devos Barlem, Pablo } \\
\text { Henrique Schmitt, Flávia Regina } \\
\text { Souza Ramos, Betina Hörner } \\
\text { Schlindwein Meirelles. }\end{array}$ & $\begin{array}{l}\text { Artigo } \\
\text { Original }\end{array}$ & Enfermagem & $\begin{array}{l}\text { Identificar as estratégias de enfrentamento dos } \\
\text { enfermeiros de serviços de oncologia, na alta } \\
\text { complexidade hospitalar, diante do cuidado a pessoa } \\
\text { com câncer. }\end{array}$ \\
\hline 2016 & $\begin{array}{l}\text { A Espiritualidade na } \\
\text { arte do Cuidar: } \\
\text { experiência do idoso } \\
\text { hospitalizado } \\
\text { câncer. }\end{array}$ & $\begin{array}{l}\text { Josenilda Pedreira da Silva Alves, } \\
\text { Maria de Fátima Correa Paula }\end{array}$ & $\begin{array}{l}\text { Artigo } \\
\text { Original }\end{array}$ & Enfermagem & $\begin{array}{l}\text { Desvendar o significado da espiritualidade no } \\
\text { enfrentamento } \\
\text { do câncer e delinear a relevância do serviço de saúde } \\
\text { em oferecer uma assistência espiritual como } \\
\text { suporte durante a internação hospitalar. }\end{array}$ \\
\hline 2016 & $\begin{array}{l}\text { Indicadores sensíveis } \\
\text { aos cuidados de } \\
\text { enfermagem na pessoa } \\
\text { em situação oncológica: } \\
\text { revisão sistemática da } \\
\text { literatura }\end{array}$ & $\begin{array}{l}\text { Ana Filipa Ramos, César Fonseca, } \\
\text { Inês Coelho, Sofia Guia, Vítor } \\
\text { Santos }\end{array}$ & $\begin{array}{l}\text { Revisão } \\
\text { Sistemática }\end{array}$ & Enfermagem & $\begin{array}{l}\text { Identificar quais os indicadores sensíveis ao cuidado de } \\
\text { enfermagem na pessoa em situação oncológica, com } 45 \\
\text { anos ou mais. }\end{array}$ \\
\hline 2015 & $\begin{array}{lr}\text { (In)satisfação } & \text { dos } \\
\text { profissionais de } & \text { saúde } \\
\text { no trabalho } & \text { em } \\
\text { oncologia }\end{array}$ & $\begin{array}{l}\text { Maiara Bordignon, Lucimare } \\
\text { Ferraz, Carmem Lúcia Colomé } \\
\text { Beck, Simone Coelho Amestoy, } \\
\text { Letícia de Lima Trindade. }\end{array}$ & $\begin{array}{l}\text { Artigo } \\
\text { Original }\end{array}$ & Multidisciplinar & $\begin{array}{l}\text { Identificar as fontes de satisfação e insatisfação no } \\
\text { trabalho dos profissionais de saúde que atuam na } \\
\text { oncologia. }\end{array}$ \\
\hline 2015 & $\begin{array}{l}\text { Hospitalizações por } \\
\text { neoplasias em idosos no } \\
\text { âmbito do Sistema } \\
\text { Único de saúde na } \\
\text { paraíba/brasil. }\end{array}$ & $\begin{array}{l}\text { Maria Yvone Queiroz Formiga, } \\
\text { Ana Elisa Vieira Fernandes Silva, } \\
\text { Mirella Bezerra de Lima Silva, } \\
\text { Raiara Carvalho Vieira, Miéllio } \\
\text { Melo Galdino. }\end{array}$ & $\begin{array}{l}\text { Artigo } \\
\text { Original }\end{array}$ & Medicina & $\begin{array}{l}\text { Avaliar a frequência de hospitalizações de pacientes } \\
\text { idosos com diagnóstico principal de câncer no sistema } \\
\text { público de saúde do Estado da Paraíba, Brasil, entre } \\
2008 \text { e } 2013 \text {, e verificar as localizações mais frequentes } \\
\text { da doença e sua } \\
\text { mortalidade hospitalar. }\end{array}$ \\
\hline 2015 & $\begin{array}{lr}\text { Acolhimento } & \text { de } \\
\text { enfermagem } & \text { em } \\
\text { oncologia: } & \\
\text { enfrentamento } & \text { da } \\
\text { doença pelos pacientes }\end{array}$ & $\begin{array}{l}\text { Glaucia Costa Machado, Ermyro } \\
\text { Thiago Rios do Ouro, Flávia } \\
\text { Pontes Guerra de Santana }\end{array}$ & $\begin{array}{l}\text { Artigo } \\
\text { Original }\end{array}$ & Enfermagem & $\begin{array}{l}\text { Investigar como tem se dado o acolhimento de } \\
\text { enfermagem a pacientes oncológicos e quais os } \\
\text { reflexos evidenciados no } \\
\text { enfrentamento da doença pelos acometidos em Feira de } \\
\text { Santana-BA. }\end{array}$ \\
\hline
\end{tabular}




\begin{tabular}{|c|c|c|c|c|c|}
\hline & $\begin{array}{l}\text { em Feira de Santana- } \\
\text { BA. }\end{array}$ & & & & \\
\hline 2015 & $\begin{array}{l}\text { Assistência de } \\
\text { enfermagem ao paciente } \\
\text { com dor oncológico }\end{array}$ & $\begin{array}{l}\text { Daniela Alves Borges, Seni Alves } \\
\text { de Oliveira. }\end{array}$ & $\begin{array}{l}\text { Revisão } \\
\text { bibliográfica } \\
\text { narrativa. } \\
\end{array}$ & Enfermagem & $\begin{array}{l}\text { Identificar a produção do conhecimento sobre a } \\
\text { enfermagem e a dor do paciente com câncer. }\end{array}$ \\
\hline 2015 & $\begin{array}{l}\text { A percepção da equipe } \\
\text { de enfermagem acerca } \\
\text { do atendimento } \\
\text { prestado ao idoso } \\
\text { Hospitalizado com dor. }\end{array}$ & $\begin{array}{l}\text { Arlete Eli Kunz da Costa, Noeli } \\
\text { Juarez Ferla, Rodrigo Bachi, } \\
\text { Claudete Moreschi, Luis Felipe } \\
\text { Pissaia. }\end{array}$ & $\begin{array}{l}\text { Artigo } \\
\text { Original }\end{array}$ & Enfermagem & $\begin{array}{l}\text { Conhecer a percepção da equipe de enfermagem de um } \\
\text { hospital de pequeno } \\
\text { porte do Rio Grande do Sul acerca do atendimento } \\
\text { prestado ao idoso hospitalizado com dor. }\end{array}$ \\
\hline 2015 & $\begin{array}{l}\text { O burnout nos } \\
\text { enfermeiros que } \\
\text { trabalham em cuidados } \\
\text { paliativos: uma revisão } \\
\text { sistemática } \\
\end{array}$ & $\begin{array}{l}\text { Iolanda Rosado Mendes da Silva, } \\
\text { Felismina Mendes. }\end{array}$ & $\begin{array}{l}\text { Revisão } \\
\text { Sistemática }\end{array}$ & Enfermagem & $\begin{array}{l}\text { A analisar a atual literatura sobre o burnout nos } \\
\text { enfermeiros de cuidados paliativos. }\end{array}$ \\
\hline 2015 & $\begin{array}{l}\text { O enfermeiro que } \\
\text { atua em unidades } \\
\text { hospitalares } \\
\text { oncológicas: perfil e } \\
\text { capacitação } \\
\text { profissional. }\end{array}$ & $\begin{array}{l}\text { Fabiana Cristina dos } \\
\text { Santos, Silvia Helena } \\
\text { Henriques Camelo, Ana } \\
\text { Maria Laus, Laura } \\
\text { Andrian Leal }\end{array}$ & $\begin{array}{l}\text { Revisão } \\
\text { Integrativa }\end{array}$ & Enfermagem & $\begin{array}{l}\text { Identificar o perfil do enfermeiro que atua em unidades } \\
\text { hospitalares oncológicas. }\end{array}$ \\
\hline 2015 & $\begin{array}{l}\text { As reações do familiar } \\
\text { acompanhante de idosos } \\
\text { hospitalizados frente às } \\
\text { situações de estresse. }\end{array}$ & $\begin{array}{l}\text { Carla Lube de Pinho Chibante, } \\
\text { Fátima Helena do Espírito Santo, } \\
\text { Alessandra Cristina de Oliveira } \\
\text { Aquino. }\end{array}$ & $\begin{array}{l}\text { Artigo } \\
\text { Original }\end{array}$ & Enfermagem & $\begin{array}{l}\text { Caracterizar o familiar acompanhante de idosos } \\
\text { hospitalizados; descrever as reações do familiar } \\
\text { acompanhante de idosos hospitalizados frente às } \\
\text { situações de estresse e discutir as possibilidades de } \\
\text { intervenção do enfermeiro ao familiar acompanhante } \\
\text { desses idosos. }\end{array}$ \\
\hline 2015 & $\begin{array}{lrr}\text { Estratégias } & & \text { para } \\
\text { humanizar } & \text { o } & \text { cuidado } \\
\text { com } \quad \text { o } & \text { idoso } \\
\end{array}$ & $\begin{array}{l}\text { Kalina Coeli Costa de Oliveira } \\
\text { Dias, Maria Emília Limeira Lopes, } \\
\text { Inacia Sátiro Xavier de França, }\end{array}$ & $\begin{array}{l}\text { Artigo } \\
\text { Original }\end{array}$ & Enfermagem & $\begin{array}{l}\text { Investigar as estratégias que enfermeiros assistenciais } \\
\text { empregam para assistir o idoso hospitalizado. }\end{array}$ \\
\hline
\end{tabular}




\begin{tabular}{|c|c|c|c|c|c|}
\hline & $\begin{array}{l}\text { hospitalizado: estudo } \\
\text { com enfermeiros } \\
\text { assistenciais. }\end{array}$ & $\begin{array}{l}\text { Patricia Serpa de Souza Batista, } \\
\text { Jaqueline Brito Vidal Batista, } \\
\text { Francisco Stélio de Sousa. }\end{array}$ & & & \\
\hline 2015 & $\begin{array}{l}\text { Produção científica } \\
\text { sobre a pessoa idosa em } \\
\text { cuidados paliativos: } \\
\text { estudo bibliométrico. }\end{array}$ & $\begin{array}{l}\text { Marcella Costa Souto Duarte, } \\
\text { Solange Fátima Geraldo da Costa, } \\
\text { Gilvânia Smith da Nóbrega } \\
\text { Morais3, Jael Rúbia Figueiredo de } \\
\text { Sá França , Maria Andréa } \\
\text { Fernandes, Maria Emília Limeira } \\
\text { Lopes. }\end{array}$ & $\begin{array}{l}\text { Revisão } \\
\text { Bibliométrica }\end{array}$ & Enfermagem & $\begin{array}{l}\text { Caracterizar a produção científica divulgada em } \\
\text { periódicos online sobre a pessoa idosa em cuidados } \\
\text { paliativos. }\end{array}$ \\
\hline 2014 & 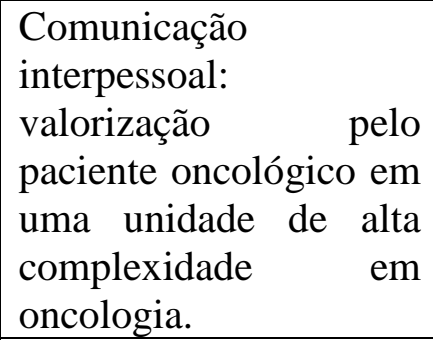 & $\begin{array}{lrr}\text { Cibele } & \text { Siqueira } & \text { Nascimento } \\
\text { Rennó, Claudinei } & \text { José Gomes } \\
\text { Campos. } & & \\
\end{array}$ & $\begin{array}{l}\text { Artigo } \\
\text { Original }\end{array}$ & Enfermagem & $\begin{array}{l}\text { Analisar a percepção dos clientes de um ambulatório de } \\
\text { oncologia acerca da comunicação interpessoal } \\
\text { profissional-cliente. }\end{array}$ \\
\hline 2014 & $\begin{array}{l}\text { Avaliação da } \\
\text { capacidade funcional do } \\
\text { paciente } \\
\text { oncogeriátrico } \\
\text { hospitalizado } \\
\end{array}$ & $\begin{array}{l}\text { Esdras Edgar Batista Pereira, } \\
\text { Edilene do Socorro Nascimento } \\
\text { Falcão Sarges, Nadia Barreto dos } \\
\text { Santos. }\end{array}$ & $\begin{array}{l}\text { Artigo } \\
\text { Original }\end{array}$ & Multidisciplinar & $\begin{array}{l}\text { Avaliar a capacidade funcional e o desempenho dos } \\
\text { sistemas funcionais de idosos com diagnóstico de } \\
\text { câncer. }\end{array}$ \\
\hline 2014 & $\begin{array}{l}\text { O cuidado ao paciente } \\
\text { oncológico }\end{array}$ & $\begin{array}{l}\text { Jonatan Fernando Beschaira } \\
\text { Bueno, Cleci Lourdes Schmidt } \\
\text { Piovesan Rosanelli, Jéssica } \\
\text { Eduarda Gomes, Marli Maria } \\
\text { Loro, Roberto Farias Sobrinho. }\end{array}$ & $\begin{array}{l}\text { Ensaio } \\
\text { Teórico }\end{array}$ & Enfermagem & $\begin{array}{l}\text { Fazer uma reflexão sobre as repercussões do câncer na } \\
\text { vida do familiar cuidador. }\end{array}$ \\
\hline 2014 & $\begin{array}{l}\text { De um lado ao outro: o } \\
\text { que é essencial? } \\
\text { Percepção dos pacientes }\end{array}$ & \begin{tabular}{|lcr} 
Bruna & Antenussi & Munhoz, \\
Henrique & Soares Paiva, & Beatrice \\
Martinez & Zugaib & Abdalla, \\
\end{tabular} & $\begin{array}{l}\text { Artigo } \\
\text { Original }\end{array}$ & Medicina & $\begin{array}{l}\text { Avaliar a percepção dos pacientes oncológicos e de } \\
\text { seus cuidadores no início da abordagem diagnóstica e } \\
\text { terapêutica, e durante os cuidados paliativos. }\end{array}$ \\
\hline
\end{tabular}




\begin{tabular}{|c|c|c|c|c|c|}
\hline & $\begin{array}{l}\text { oncológicos e de seus } \\
\text { cuidadores ao iniciar o } \\
\text { tratamento oncológico e } \\
\text { em cuidados paliativos. }\end{array}$ & $\begin{array}{l}\text { Guilherme Zaremba, Andressa } \\
\text { Macedo Paiva Rodrigues, Mayra } \\
\text { Ribeiro Carretti, Camila Ribeiro de } \\
\text { Arruda Monteiro, Aline Zara, } \\
\text { Jussara Oliveira Silva, Widner } \\
\text { Baptista Assis, Luciana Campi } \\
\text { Auresco, Leonardo Lopes Pereira, } \\
\text { Adriana Braz del Giglio, Ana } \\
\text { Claudia de Oliveira Lepori, Damila } \\
\text { Cristina Trufelli, Auro del Giglio. }\end{array}$ & & & \\
\hline 2014 & $\begin{array}{l}\text { Percepção da equipe de } \\
\text { enfermagem } \\
\text { cuidadonte aos } \\
\text { oncológicos: } \begin{aligned} \text { paliativos } \\
\text { fenomenológico }\end{aligned}\end{array}$ & $\begin{array}{l}\text { Waleska Christina Brandão Pereira } \\
\text { da Silva, Rose Mary Costa Rosa } \\
\text { Andrade Silva, Eliane Ramos } \\
\text { Pereira, Marcos Andrade Silva, } \\
\text { Aline Miranda da Fonseca Marins, } \\
\text { Marta Sauthier. }\end{array}$ & $\begin{array}{l}\text { Artigo } \\
\text { Original }\end{array}$ & Enfermagem & $\begin{array}{l}\text { Compreender a percepção da equipe de enfermagem } \\
\text { frente ao cuidado paliativo em oncologia a partir do } \\
\text { referencial fenomenológico em Merleau-Ponty; indicar } \\
\text { as implicações desta percepção na práxis da } \\
\text { Enfermagem. }\end{array}$ \\
\hline 2014 & $\begin{array}{l}\text { Atenção da enfermagem } \\
\text { à saúde do idoso: uma } \\
\text { revisão integrativa. }\end{array}$ & $\begin{array}{l}\text { Sara Cordeiro Eloia, Eliany Nazaré } \\
\text { Oliveira, Suzana Mara Cordeiro } \\
\text { Eloia, Roberlandia Evangelista } \\
\text { Lopes }\end{array}$ & $\begin{array}{l}\text { Artigo } \\
\text { Original }\end{array}$ & Enfermagem & $\begin{array}{l}\text { Analisar a produção científica na área da atenção à } \\
\text { saúde do idoso pela enfermagem. }\end{array}$ \\
\hline 2014 & $\begin{array}{lr}\text { Consulta } & \text { de } \\
\text { enfermagem: } & \text { avaliação } \\
\text { da adesão ao } & \text { ao } \\
\text { autocuidado dos } \\
\text { pacientes submetidos à } \\
\text { radioterapia. }\end{array}$ & $\begin{array}{l}\text { Karla Biancha Silva de AndradeI, } \\
\text { Anna Clara Lima FranczI, } \\
\text { Marianne dos Santos GrellmannIII, } \\
\text { Priscila Cortez Belchior, Josiana } \\
\text { Araujo de Oliveira,Dayse do } \\
\text { Nascimento Wassita. }\end{array}$ & $\begin{array}{l}\text { Artigo } \\
\text { Original }\end{array}$ & Enfermagem & $\begin{array}{l}\text { Avaliar a adesão dos pacientes às orientações } \\
\text { fornecidas na consulta de enfermagem em radioterapia. }\end{array}$ \\
\hline 2014 & $\begin{array}{l}\text { Fadiga em idosos em } \\
\text { tratamento } \\
\text { quimioterápico. }\end{array}$ & \begin{tabular}{lrr|} 
Thalytarastina & Cristano, \\
SchlosserI, & Maria & Filomena \\
Ceolim. & & \\
\end{tabular} & $\begin{array}{l}\text { Artigo } \\
\text { Original }\end{array}$ & Enfermagem & $\begin{array}{l}\text { Identificar a presença e intensidade da fadiga em } 140 \\
\text { idosos (média de idade } 69,8 \text { anos, } 52,1 \% \text { do sexo } \\
\text { feminino) com diagnóstico de câncer, em tratamento } \\
\text { quimioterápico ambulatorial. }\end{array}$ \\
\hline
\end{tabular}




\begin{tabular}{|c|c|c|c|c|c|}
\hline 2014 & $\begin{array}{l}\text { Vivências emocionais } \\
\text { de } \quad \text { pacientes } \\
\text { oncológicos submetidos } \\
\text { à quimioterapia. }\end{array}$ & $\begin{array}{l}\text { Catarina Possenti Sette, Silvia } \\
\text { Mayumi Obana Gradvohl }\end{array}$ & $\begin{array}{l}\text { Artigo } \\
\text { Original }\end{array}$ & Psicologia & $\begin{array}{l}\text { Apresentar uma visão sobre Psicologia no contexto da } \\
\text { Oncologia, bem como relatar o trabalho realizado a } \\
\text { partir de um campo de estágio da Psicologia Hospitalar, } \\
\text { desenvolvido em uma clínica particular de um } \\
\text { município do interior de São Paulo. }\end{array}$ \\
\hline 2014 & $\begin{array}{l}\text { Participação do familiar } \\
\text { nos cuidados paliativos } \\
\text { oncológicos no contexto } \\
\text { hospitalar: perspectiva } \\
\text { de enfermeiros. }\end{array}$ & $\begin{array}{l}\text { Marcelle Miranda da Silva, } \\
\text { Lorhanna da Silva Lima. }\end{array}$ & $\begin{array}{l}\text { Artigo } \\
\text { Original }\end{array}$ & Enfermagem & $\begin{array}{l}\text { Compreender a perspectiva de enfermeiros acerca da } \\
\text { participação do familiar na hospitalização em cuidados } \\
\text { paliativos } \\
\text { oncológicos e analisar as estratégias de cuidado de } \\
\text { enfermagem para atendimento das necessidades } \\
\text { daquele. }\end{array}$ \\
\hline 2013 & $\begin{array}{l}\text { Repercussões do } \\
\text { Tratamento } \\
\text { quimioterápico para a } \\
\text { pessoa idosa. }\end{array}$ & $\begin{array}{l}\text { Michele Antunes, } \\
\text { Vera Lucia Fortunato Fortes, } \\
\text { Daniela Ramos Oliveira, } \\
\text { Débora Corso, } \\
\text { Luiz Antonio Bettinelli, } \\
\text { Dalva Maria Pomatti. }\end{array}$ & $\begin{array}{l}\text { Artigo } \\
\text { Original }\end{array}$ & Enfermagem & $\begin{array}{l}\text { Analisar como o idoso com câncer expressa sua } \\
\text { condição de realizar quimioterapia ambulatorial em um } \\
\text { hospital geral do norte do Rio Grande do Sul. }\end{array}$ \\
\hline 2013 & $\begin{array}{l}\text { Perfil do idoso em } \\
\text { tratamento } \\
\text { radioterápico }\end{array}$ & $\begin{array}{l}\text { Caroline de Leon Linck, Celmira } \\
\text { Lange, Juliana Zillmer, Alitéia } \\
\text { Santiago Dilélio, } \\
\text { Eda Schwartz. }\end{array}$ & $\begin{array}{l}\text { Artigo } \\
\text { Original }\end{array}$ & Enfermagem & $\begin{array}{l}\text { Conhecer o perfil dos idosos em tratamento } \\
\text { radioterápico de um Centro Regional de Radioterapia } \\
\text { da Região Sul do } \\
\text { Brasil. }\end{array}$ \\
\hline 2013 & $\begin{array}{l}\text { Família do idoso com } \\
\text { câncer: vivenciando as } \\
\text { dificuldades }\end{array}$ & $\begin{array}{l}\text { Bruna Moretti Luchesi, Tábatta } \\
\text { Renata Pereira de Brito, Liliam } \\
\text { Léia Biazin Silveira, Noeli } \\
\text { Marchioro Liston Andrade } \\
\text { Ferreira, Giselle Dupas, Carmem } \\
\text { Lúcia Alves Filizola }\end{array}$ & $\begin{array}{l}\text { Artigo } \\
\text { Original }\end{array}$ & Enfermagem & $\begin{array}{l}\text { Investigar como o cuidado ao idoso com câncer tem } \\
\text { sido vivenciado pela família brasileira. }\end{array}$ \\
\hline 2013 & $\begin{array}{lr}\text { Estratégias } & \text { de } \\
\text { enfrentamento usadas } \\
\text { por enfermeiros ao }\end{array}$ & $\begin{array}{l}\text { Anna Maria de Oliveira Salimena, } \\
\text { Simone de Rezende Teixeira, } \\
\text { Thaís Vasconselos Amorim, }\end{array}$ & $\begin{array}{l}\text { Artigo } \\
\text { Original }\end{array}$ & Enfermeiro & $\begin{array}{l}\text { Conhecer as estratégias que os enfermeiros utilizam } \\
\text { para lidar com os abalos psicoemocionais advindos do } \\
\text { processo de cuidado de pacientes oncológicos. }\end{array}$ \\
\hline
\end{tabular}




\begin{tabular}{|c|c|c|c|c|c|}
\hline & $\begin{array}{l}\text { cuidar de pacientes } \\
\text { oncológicos. }\end{array}$ & $\begin{array}{l}\text { Andyara do Carmo Pinto Coelho } \\
\text { Paiva, Maria Carmen Simões } \\
\text { Cardoso de Melo. }\end{array}$ & & & \\
\hline 2013 & $\begin{array}{l}\text { O vivido dos } \\
\text { enfermeiros no cuidado } \\
\text { ao paciente oncológico. }\end{array}$ & $\begin{array}{l}\text { Anna Maria de Oliveira Salimena, } \\
\text { Simone de Rezende Teixeira, } \\
\text { Thaís Vasconselos Amorim, } \\
\text { Andyara do Carmo Pinto Coelho } \\
\text { Paiva, Maria Carmen Simões } \\
\text { Cardoso de Melo. }\end{array}$ & $\begin{array}{l}\text { Artigo } \\
\text { Original }\end{array}$ & Enfermeiro & $\begin{array}{l}\text { Conhecer as percepções e sentimentos de enfermeiros } \\
\text { de um hospital oncológico de referência em diagnóstico } \\
\text { e tratamento de pacientes com câncer na Zona da Mata } \\
\text { Mineira. }\end{array}$ \\
\hline 2013 & $\begin{array}{l}\text { O morrer e a morte de } \\
\text { idosos hospitalizados na } \\
\text { ótica de profissionais de } \\
\text { enfermagem. }\end{array}$ & $\begin{array}{l}\text { Juliane Elis Both, Marinês } \\
\text { Tambara Leite, } \\
\text { Leila Mariza Hildebrandt, Jacinta } \\
\text { Spies, } \\
\text { Luiz Anildo Anacleto da Silva, } \\
\text { Margrid Beuter }\end{array}$ & $\begin{array}{l}\text { Artigo } \\
\text { Original }\end{array}$ & Enfermagem & $\begin{array}{l}\text { Analisar a percepção de profissionais de enfermagem } \\
\text { acerca da morte de idosos } \\
\text { Hospitalizados. }\end{array}$ \\
\hline 2013 & $\begin{array}{l}\text { Obstáculos no } \text { cuidado } \\
\text { às pessoas idosas } \\
\text { hospitalizadas: } \\
\text { percepções } \\
\text { enfermeiros(as). }\end{array}$ & $\begin{array}{l}\text { João Tavares, Alcione Leite da } \\
\text { Silva }\end{array}$ & $\begin{array}{l}\text { Artigo } \\
\text { Original }\end{array}$ & Enfermagem & $\begin{array}{l}\text { Conhecer as percepções de enfermeiros(as) acerca do } \\
\text { cuidado às pessoas idosas hospitalizadas e dos } \\
\text { obstáculos enfrentados para desenvolver um cuidado de } \\
\text { boa qualidade. }\end{array}$ \\
\hline 2013 & $\begin{array}{l}\text { A enfermagem } \\
\text { oncológica frente ao } \\
\text { câncer em pacientes } \\
\text { idosos. }\end{array}$ & $\begin{array}{l}\text { Armelita Elenice Vianna, Cintia da } \\
\text { Silva Marconato, Lucélia Gindri, } \\
\text { Micheline Raquel Beneton, } \\
\text { Suzinara Beatriz Soares de Lima, } \\
\text { Viviani Viero. }\end{array}$ & $\begin{array}{l}\text { Pesquisa } \\
\text { Documental }\end{array}$ & Enfermeiro & $\begin{array}{l}\text { Investigar os tipos de câncer de maior incidência nos } \\
\text { pacientes a partir dos } 60 \text { anos de idade, atendidos no } \\
\text { Ambulatório de Quimioterapia do Hospital } \\
\text { Universitário de Santa Maria } \\
\text { (HUSM). }\end{array}$ \\
\hline 2012 & $\begin{array}{l}\text { O olhar da enfermagem } \\
\text { sobre as práticas de } \\
\text { cuidado de famílias } \\
\text { rurais à pessoa com } \\
\text { câncer }\end{array}$ & $\begin{array}{l}\text { Juliana Graciela Vestena Zillmer, } \\
\text { Eda Schwartz, Rosani Manfrin } \\
\text { Muniz }\end{array}$ & $\begin{array}{l}\text { Artigo } \\
\text { Original }\end{array}$ & Enfermagem & $\begin{array}{l}\text { Identificar as } \\
\text { Práticas de cuidados das famílias rurais que } \\
\text { vivenciam o cuidar da pessoa com câncer. }\end{array}$ \\
\hline
\end{tabular}




\begin{tabular}{|c|c|c|c|c|c|}
\hline 2012 & $\begin{array}{l}\text { A ética do enfermeiro } \\
\text { no cuidado a pacientes } \\
\text { idosos sem } \\
\text { possibilidades } \\
\text { terapêuticas de cura }\end{array}$ & $\begin{array}{l}\text { Jéssyka Cibelly Minervina da } \\
\text { Costa Silva, Lariça Cândido da } \\
\text { Silva, Cleane Rosa da Silva, } \\
\text { Thayana Jovino Oliveira, Leila de } \\
\text { Cássia Tavares da Fonseca. }\end{array}$ & $\begin{array}{l}\text { Artigo } \\
\text { Original }\end{array}$ & Enfermagem & $\begin{array}{l}\text { Compreender a importância ética do enfermeiro no } \\
\text { cuidado a pacientes idosos sem possibilidades } \\
\text { terapêuticas de cura. }\end{array}$ \\
\hline 2012 & $\begin{array}{l}\text { Os cuidados de } \\
\text { enfermagem } \\
\text { Em feridas neoplásicas } \\
\text { Na assistência paliativa }\end{array}$ & $\begin{array}{l}\text { Rafaela Mouta Aguiar, Gloria } \\
\text { Regina C. da Silva. }\end{array}$ & $\begin{array}{l}\text { Artigo } \\
\text { Original }\end{array}$ & Enfermagem & $\begin{array}{l}\text { Propor um cuidado humanizado, prestando uma } \\
\text { assistência mais efetiva e dinâmica, que minimize o } \\
\text { desconforto, a dor, e os transtornos psicossociais que } \\
\text { podem ser gerados pelas feridas oncológicas. }\end{array}$ \\
\hline 2011 & $\begin{array}{l}\text { Fragilidade no idoso: o } \\
\text { que vem } \quad \text { sendo } \\
\text { produzido } \quad \text { pela } \\
\text { enfermagem }\end{array}$ & $\begin{array}{l}\text { Caroline de Leon Lincka, Maria da } \\
\text { Graça Oliveira Crossettib }\end{array}$ & $\begin{array}{l}\text { Artigo } \\
\text { Original }\end{array}$ & Enfermagem & $\begin{array}{l}\text { Identificar a produção científica desenvolvida pela } \\
\text { enfermagem frente à fragilidade no idoso, a partir de } \\
\text { uma revisão integrativa da literatura, realizada nas } \\
\text { bases Scientific Electronic Library Online (SciELO), } \\
\text { Literatura Latino Americana e do Caribe em Ciências } \\
\text { da Saúde (LILACS) e Scopus. }\end{array}$ \\
\hline 2011 & $\begin{array}{l}\text { Percepção } r \text { do } \\
\text { enfermeiro sobre o } \\
\text { cuidado prestado aos } \\
\text { pacientes portadores de } \\
\text { neoplasia. }\end{array}$ & $\begin{array}{l}\text { Camila Prearo; Lívia de Souza } \\
\text { Gonçalves; Marina Botelho } \\
\text { Vinhando; Sarita Lopes Menezes. }\end{array}$ & $\begin{array}{l}\text { Artigo } \\
\text { Original }\end{array}$ & Enfermagem & $\begin{array}{l}\text { Compreender o significado que o enfermeiro atribui ao } \\
\text { cuidado que dispensa ao paciente portador de } \\
\text { neoplasia, considerando os aspectos biopsicossociais e } \\
\text { a relevância do seu relacionamento terapêutico. }\end{array}$ \\
\hline 2011 & $\begin{array}{l}\text { Um olhar sobre o } \\
\text { cuidador de pacientes } \\
\text { oncológicos recebendo } \\
\text { cuidados paliativos }\end{array}$ & $\begin{array}{l}\text { Claudiane Aparecida Guimarães, } \\
\text { Marilda Emmanuel Novaes Lipp. }\end{array}$ & $\begin{array}{l}\text { Artigo } \\
\text { Original }\end{array}$ & Psicologia & $\begin{array}{l}\text { Averiguar como o cuidador principal de pacientes } \\
\text { oncológicos recebendo cuidados paliativos vivencia o } \\
\text { seu ato de cuidar e como a iminência da perda do } \\
\text { paciente afeta o seu nível de estresse. }\end{array}$ \\
\hline 2011 & $\begin{array}{lr}\text { Conhecimento } & \text { de } \\
\text { Profissionais } & \text { da } \\
\text { Enfermagem } & \text { sobre } \\
\text { Fatores que Agravam e } \\
\text { Aliviam a } & \text { Dor } \\
\text { Oncológica } & \end{array}$ & $\begin{array}{l}\text { Vanessa Souza Alves, Tamires } \\
\text { Saniely dos Santos, Maria Cristina } \\
\text { Soares Figueiredo Trezza, Regina } \\
\text { Maria dos Santos, Fernanda Silva } \\
\text { Monteiro. }\end{array}$ & $\begin{array}{l}\text { Artigo } \\
\text { Original }\end{array}$ & Enfermagem & $\begin{array}{l}\text { Avaliar o nível de conhecimento de profissionais da } \\
\text { enfermagem sobre fatores que agravam e aliviam a dor. }\end{array}$ \\
\hline
\end{tabular}




\begin{tabular}{|l|l|l|l|l|l|}
\hline 2011 & $\begin{array}{l}\text { Comunicação } \\
\text { terapêutica } \\
\begin{array}{l}\text { Enfermagem: na } \\
\text { dificuldades para o } \\
\text { cuidar de idosos com } \\
\text { câncer. }\end{array}\end{array}$ & $\begin{array}{l}\text { Aline Azevedo Peterson, Emília } \\
\text { Campos de Carvalho. }\end{array}$ & $\begin{array}{l}\text { Artigo } \\
\text { Original }\end{array}$ & $\begin{array}{l}\text { Enfermagem } \\
\text { percepção do enfermeiro, ao se prestar assistência ao } \\
\text { paciente idoso, com patologia oncológica. }\end{array}$ \\
\hline
\end{tabular}

Quadro 1 - Distribuição dos artigos (n), segundo ano, título, autores, modalidade de artigo, área da revista e objetivos das publicações selecionadas 
Os novos casos do câncer no Brasil e no mundo cresem num ritmo que acompanha a longevidade, decorrente do aumento da expectativa de vida (INCA, 2011).

Observa-se um incremento na incidência de novos casos de câncer na população idosa, estes são confirmados pelos dados de 2011 do INCA (2011): no Brasil $70 \%$ dos diagnósticos de câncer ocorrem em indivíduos acima de 60 anos, e, deste universo, perto $60 \%$ tem mais de 70 anos de idade.

Estima-se que no ano de 2030, possam ser registrados 27 milhões de novos casos de neoplasias a nível mundial, repercutindo em 75 milhões de pessoas vivendo com a doença anualmente e 17 milhões de mortes (BRASIL, 2011), sinalizando então, a necessidade de profissionais ada vez mais qualificados para dar subsídios no tratamento, reabilitação, comunicação, cura e cuidados paliativos.

Visentin e Lenardt (2010) endossam que a oncologia e o envelhecimento arrolam-se de forma direta, de talforma que o quantitativo de casos de câncer sofre um aumento proporcional à idade, isto é, quanto mais a população se torna longeva, há uma tendência no crescimento do número de casos de câncer. Além disso, o câncer produz impacto significativo na geriatria e gerontologia de todo o mundo, isto implica em repensarmos os cuidados e a implementação de novas estratégias que visem o melhor atendimento a essa parcela da população.

Os cuidados de enfermagem prestados aos pacientes oncológicos devem ser caracterizados sobretudo no que tange à idade e implicações, uma vez que cada ciclo de vida proporciona transformações fisiológicas e psíquicas. O paciente idoso, por exemplo, está fragilizado pelo natural processo do envelhecimento, além de uma perspectiva de sobrevida reduzida, o que pode intuir quanto à medo e morte. Por isso, diante de um diagnóstico de uma doença neoplásica maligna, a sua esperança se torna bem reduzida e ocorre um grau de sofrimento orgânico considerável e diferenciado (PETERSON, CARVALHO, 2011).

Considera-se que as aplicações da ciência e da tecnologia no processo de cuidar no campo da enfermagem, tem entusiasmado significativamente o exercício destes profissionais em decorrência do surgimento de dilemas éticos durante as práticas profissionais, inferindo-se, assim, acerca da importância que da bioética para com a sua tomada de decisões frente a problemas e vivências morais (MASCARENHAS E ROSA, 2010)

A equipe de enfermagem no contexto hospitalar lida com grandes encargos frente a estes pacientes, tendo como aptidão principal, prestar assistência na avaliação diagnóstica, no 
tratamento, na reabilitação, na comunicação com todos os públicos envolvidos e no atendimento aos familiares. Além disso, lida continuamente com situações de sofrimento e morte, que podem ser atenuadas por predicativos da demanda, do ambiente de trabalho e pessoais. Esse contexto de trabalho exige dos enfermeiros uma assistência com prioridade na avaliação e atribuição integrais, tanto do paciente, quanto de sua família, superando os limites da própria doença e do adoecimento (FURTADO et al., 2009; AMADOR et al., 2011).

Segundo Brito e Carvalho (2010), o respeito à individualidade, à intimidade, às crenças e valores, e o apoio no difícil momento de adoecimento por neoplasia, são, sem dúvida, diferenciais para o tratamento, afora a contribuição na diminuição do negativismo que o ambiente hospitalar possa aludir (BRITO E CARVALHO, 2010).

Indubitavelmente, o indivíduo que vivencia a hospitalização passa uma situação estressante, de mudança de rotina, privação de locomoção e, muitas vezes, de sofrimento. Características próprias da relação humana, como compaixão, atenção, zelo, expressão de afeto, demonstração de respeito e tolerância, qualificam o cuidado e a contribuição técnico específica ao atendimento do idoso, tornam-no assim, profissional com diferencial (MARTINS, SILVA, ALVIM, 2010).

Sobre as unidades de internação, Prochet e Silva (2011) salientam que são ambientes de comunicação onde as extensões verbais e não verbais guiam as relações entre os idosos internados para tratamento e a equipe de enfermagem, consentindo várias possibilidades de diálogo que podem ser captadas e decodificadas de forma consciente ou não (PROCHET E SILVA, 2011).

Deste modo, o atendimento ao idoso frágil deve ser guiado na consideração dos cuidados individualizados, sob a conexão interdisciplinar, com vistas à manutenção da identidade do idoso, incitando a sua autonomia e tomada decisão, distinguindo-o o idoso enquanto sujeito ativo em todo o seu processo de tratamento e cuidado, sendo este um dos desafios no cenário atual (HAPP, 2010).

O enfermeiro deve prover uma maior aproximação com os idosos doentes com câncer, com foco na comunicação eficaz e personificada, visando a identificação de suas necessidades e desenvolvimento de situações que melhor possam impulsionar sua qualidade de vida (PETERSON, CARVALHO, 2011).

Almeida e Aguiar (2011) endossam que os enfermeiros possuem como principal valor na orientação do cuidado prestado ao paciente idoso hospitalizado, o respeito. Neste cerne, 
considera-se que respeito envolva valores secundários e não menos importantes, tais quais, sensibilidade, igualdade, humanização, fé e dignidade (ALMEIDA E AGUIAR, 2011). A percepção que o profissional possui sobre a dor, doença e tratamento, que expectativas traz, que medos e fantasias expressas, são também fundamentais para a garantia de uma qualidade da assistência, já que a desinformação, as informações mal repassadas ou as expectativas não realistas, constituem-se como obstáculos para um melhor cuidado, levando ansiedade e sofrimento desnecessário para o paciente e seus familiares (ALVES et al., 2011).

Ao se deparar com situações em que a vida dos pacientes é permeada por limiares críticos, os profissionais devem estabelecer uma assistência baseada nos princípios éticos e agir de forma reflexiva, a fim de garantir um cuidado efetivo e humanizado (CERRI et al., 2011).

Considera-se que na forma humanista de assistência à saúde, o enfermeiro deve estar atento para ouvir objetivamente o indivíduo acometido por doença ou dor, também, identificar suas necessidades e auxiliá-lo a nas soluções a partir da ampliação das informações de sua própria atividade mental, instrumentalizando o paciente para a ação em prol de si e do tratamento (BRASIL, 2010).

Portanto, os enfermeiros precisam estar aparelhados para o cuidado com este público, considerando-o, principalmente, em sua integralidade, acolhendo-os através da escuta qualificada dos seus sentimentos e vivências, amenizando o sofrimento de conviver com a doença e com os efeitos do tratamento (MISTURA, CARVALHO, SANTOS, 2012).

Segundo Oliveira e colaboradores (2012), dispositivos como acolhimento, companhia e consolo, qualificam o relacionamento interpessoal entre enfermeiros, pacientes e familiares que vivenciam/acompanham o processo de adoecimento neoplásico/tratamento (OLIVEIRA, SANTOS, MASTROPIETRO, 2010).

Percebe-se que há favoráveis articulações, trocas e estabelecimento de objetivos comuns, favorecendo à assistência aos idosos sob o prisma da integralidade em saúde, quando do trabalho contextualizado na equipe Além disso, estes se percebem mais valorizados e respeitados em cada saber específico, também, distinguem que o trabalho torna-se mais criativo (MATOS, PIRES, GELBCKE, 2012).

\section{Considerações Finais}


Diante da crescente taxa populacional de idosos atualmente, e, mediante a necessidade do conhecimento da práxis em enfermagem a este segmento populacional, surgiu o interesse de investigar a atuação destes profissionais da saúde aos idosos usuários dos serviços hospitalares oncológicos.

Cremos que a utilidade deste, toca não somente o pesquisador e o sujeito pesquisado, mas também, a sociedade, e os demais profissionais de saúde e gerontologia. O presente estudo não se restringe ao ambiente acadêmico unicamente, uma vez que gerará informações relevantes sobre a temática e colaborará com a ampliação de publicação nos subtemas salientados, mas, também poderá orientar a sociedade em geral. Torna-se míster referir ainda, que há uma escassez de estudos nos subtemas do presente, isto é, observa-se poucas publicações.

Diante do exposto, também julga-se importante a reflexão sobre os aspectos transpessoais e multidiscipinar envolvidos no diagnóstico e tratamento do câncer, tanto sob a perspectiva dos profissionais que assistem diretamente os pacientes acometidos, quanto dos idosos assistidos pelos serviços e ainda de seus familiares.

Ademais, faz-se necessária a reflexão constante acerca da capilaridade com questões de ordem físicas, psicoafetivas/de saúde mental mais ampla e comunicacionais, e que implicam no cuidado gerontológico de forma integral. Indubitavelmente, profissionais enfermeiros e ancião tem uma relação muito intensa no lócus ILPI, lidando então com um contexto de possibilidades de vivências e vivificação de situações emocionais vastas, mesmo que, estas nem sempre incorram de maneira consciente.

Não obstante, a presente revisão de literatura sobre as práticas de promoção de saúde, por meio da assistência de enfermagem aos idosos usuários dos serviços hospitalares oncológicos, pôde contribuir para avaliações e considerações acerca do cenário, na medida que, apontou o estado da arte, as fragilidades e as perspectivas.

\section{Referências}


ALMEIDA, ABA; AGUIAR, MGG. A dimensão ética do cuidado de enfermagem ao idoso hospitalizado na perspectiva de enfermeiros. Rev. Eletr. Enf. [Internet]. 2011 jan/mar;13(1):42-9.

ALVES, VS; SANTOS, TS; TREZZA, MCSF; SANTOS, RM; MONTEIRO, FS. Conhecimento de Profissionais da Enfermagem sobre Fatores que Agravam e Aliviam a Dor Oncológica. Revista Brasileira de Cancerologia 2011; 57(2):199-206.

AMADOR, DD; GOMES, IP; COUTINHO, SED; COSTA, TNA; COLLET, N. Nurses' conceptions about pediatric oncology care training. 2011.

BRASIL, Instituto Nacional do Câncer. Estimativa 2012 - Incidência de câncer no Brasil. Rio de Janeiro; 2011.

BRASIL. Instituto Nacional do Câncer. Estimativa 2012: incidência de câncer no Brasil/Instituto Nacional de Câncer. Rio de Janeiro: INCA; 2011.

BRASIL. MINISTÉRIO DA SAÚDE. INSTITUTO NACIONAL DE CÂNCER (INCA). Disponível em: <http:www.inca.gov.br>. Acesso em:01 de abril de 2011.

BRASIL. Ministério da Saúde. Instituto Nacional do Câncer. Coordenação Geral de Gestão Assistencial. Coordenação de Educação. Comunicação de notícias difíceis: compartilhando desafios na atenção à saúde. Rio de Janeiro: INCA; 2010; 206 p.

BRITO, NTG; CARVALHO, R. A humanização segundo pacientes oncológicos com longo período de internação. Einstein. 2010; 8(2pt1):221-7.

CERRI, A; ROEHRS, A; CROZETA, K; SARQUIS, LMM; PALU, L. Problemas éticos no cuidado ao paciente crítico. Cogitare Enferm. 2011;16(3):463-70.

FURTADO, SB; LÔBO, AS; SANTOS, MCL; SILVA, APS; FERNANDES, AFC. Understanding feelings about breast cancer: nurses' report. Rev RENE [Internet]. 2009.

GIRO, A; PAÚL, C. Envelhecimento Sensorial, Declínio Cognitivo e Qualidade de Vida no Idoso com Demência. Actas de Gerontologia. v.1, n.1, p.1-10, 2013.

HAPP, MB. Individualized care for frail older adults: challenges for health care reform in acute and critical care. Geriatr Nurs. 2010;31(1):63-5.

INSTITUTO BRASILEIRO DE GEOGRAFIA E ESTATÍSTICA. Sinopse do censo demográfico. 2010 [Internet].

MARTINS, PAF; SILVA, DC; ALVIM, NAT. Tipologia de cuidados de enfermagem segundo clientes hospitalizados: encontro das dimensões técnico-científica e expressiva. Rev Gaúcha Enferm.2010;31(1):143-50.

MASCARENHAS, NB; ROSA, DOS. Bioethics and nursing formal education: a necessary interface. Enferm [Internet]. 2010

MATOS, E; PIRES, DEP; GELBCKE, FL. Implicações da interdisciplinaridade na organização do trabalho da enfermagem: estudo em equipe de cuidados paliativos. Rev Eletrônica Enferm [internet]. 2012. 
MISTURA, C; CARVALHO, MFAA; SANTOS, VEP. Mulheres mastectomizadas: vivências frente ao câncer de mama. Rev Enferm UFSM. 2012; 1(3):351-59.

MOREIRA, M.A.D.M; LUSTOSA, A.M; DUTRA, F; BARROS, E.O; BATISTA, J.B.V; DUARTE, .C.S. Políticas públicas de humanização: revisão integrativa da literatura. Ciência \& Saúde Coletiva, 20(10):3231-3242, 2015.

OLIVEIRA, EA; SANTOS, MA; MASTROPIETRO, AP. Apoio psicológico na terminalidade: ensinamentos para a vida. Psicol. estud. 2010;15(2):235-44.

PETERSON, AA; CARVALHO, EC. Comunicação terapêutica na Enfermagem: dificuldades para o cuidar de idosos com câncer. Rev Bras Enferm, Brasília 2011 jul-ago; 64(4): 692-7.

PINTO, MH; CRUZ, MF; CESARINO, CB; PEREIRA, APS; RIBEIRO, RCHM; BECCARIA, LM. O cuidado de enfermagem ao paciente oncológico fora de possibilidade de cura: percepção de um grupo de profissionais. Cogitare enferm. 2011;16(4):647-53.

PROCHET, TC; SILVA, MJP. Percepção do idoso dos comportamentos afetivos expressos pela equipe de enfermagem. Esc Anna Nery Rev Enferm. 2011;15(4):784-90.

RENNÓ, SCN; CAMPOS, CJG. Comunicação interpessoal: valorização pelo paciente oncológico em uma unidade de alta complexidade em oncologia. Rev Min Enferm. 2014 jan/mar; 18(1): 106115.

SALIMENA, AMO; TEIXEIRA, SRT; AMORIM, TV; PAIVA, ACPC; MELO, MCSC. O vivido dos enfermeiros no cuidado ao paciente oncológico. Cogitare Enferm. 2013 Jan/Mar; 18(1):142-7.

SECRETARIA EXECUTIVA, Ministério da Saúde. Morbidade hospitalar do SUS: por local de internação no ano de 2007. Brasília (Brasil): Ministério da Saúde; 2008.

VISENTIN, A; LENARDT, M. H. O itinerário terapêutico: história oral de idosos com câncer. Acta paul. enferm. [online]. 2010, vol.23, n.4, pp. 486-492.

\section{Como citar este artigo (Formato ABNT):}

MONTEIRO, Andreane Natália P. DE; BATISTA, Maria Amanda L.;PEREIRA, Juliane da Silva; BARBOSA, Luciclaudio da S.; LEITE-SALGUEIRO, Claudia Daniele B; LOBATO, Luzineide. A Práxis do Enfermeiro na Assistência Prestada à Saúde do Idoso Usuário do Serviço Hospitalar Oncológico. Id on Line Rev.Mult. Psic., 2018, vol.12, n.41, p.225-243. ISSN: 1981-1179.

Recebido: 07/06/2018

Aceito 14/06/2018 